\title{
Self-consistent mode-coupling theory for the viscosity of rodlike polyelectrolyte solutions
}

\author{
Kunimasa Miyazaki ${ }^{\mathrm{a})}$ \\ Department of Chemistry, Columbia University, New York, New York 10027 \\ Biman Bagchi \\ Solid State and Structural Chemistry Unit, Indian Institute of Science, Bangalore 560 012, India \\ Arun Yethiraj ${ }^{\text {b) }}$ \\ Theoretical Chemistry Institute and Department of Chemistry, University of Wisconsin, Madison, \\ Wisconsin 53706
}

\begin{abstract}
A self-consistent mode-coupling theory is presented for the viscosity of solutions of charged rodlike polymers. The static structure factor used in the theory is obtained from polymer integral equation theory; the Debye-Hückel approximation is inadequate even at low concentrations. The theory predicts a nonmonotonic dependence of the reduced excess viscosity $\eta_{R}$ on concentration from the behavior of the static structure factor in polyelectrolyte solutions. The theory predicts that the peak in $\eta_{R}$ occurs at concentrations slightly lower than the overlap threshold concentration, $c^{*}$. The peak height increases dramatically with increasing molecular weight and decreases with increased concentrations of added salt. The position of the peak, as a function of concentration divided by $c^{*}$, is independent of salt concentration or molecular weight. The predictions can be tested experimentally.
\end{abstract}

\section{INTRODUCTION}

Polyelectrolyte solutions are widely considered to be one of the least understood substances in polymer science. ${ }^{1}$ There are several features of these solutions that make them rather complex. For one, the long-ranged nature of the electrostatic interactions results in long-ranged correlations even in dilute solutions. In addition, polymer conformations are very sensitive to concentration and ionic strength because the electrostatic interactions compete with short-ranged "hydrophobic" interactions. This complex static behavior is accompanied by very interesting dynamical behavior of polyelectrolyte solutions. In this work, we present a theoretical study of the viscosity of dilute and semidilute rodlike polyelectrolyte solutions using a liquid state approach.

The viscosity of polyelectrolyte solutions displays an interesting "anomalous" concentration dependence at low polymer concentrations. ${ }^{2,3}$ The quantity that is normally discussed is the reduced viscosity $\eta_{R}$ defined as $\eta_{R}$ $\equiv\left(\eta-\eta_{0}\right) /\left(\eta_{0} c_{p}\right)$, where $\eta$ is the viscosity of the solution, $\eta_{0}$ is the viscosity of the solvent in the absence of the polymer, and $c_{p}$ is the (monomer) concentration of polymers. Experiments show that $\eta_{R}$ displays a sharp peak at low polyelectrolyte concentrations for a variety of different solutions. This anomalous concentration dependence of viscosity of dilute polyelectrolyte solutions has been the focus of attention for over 50 years and, although there have been many theories that address the problem, it is not considered to be well understood. ${ }^{1,4,5}$ In fact, as discussed eloquently by Forster

\footnotetext{
a)Electronic mail: km2233@columbia.edu

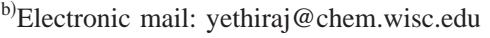

and Schmidt, ${ }^{1}$ this problem has witnessed some grave mistakes, causing much confusion.

A contributing factor to the above-mentioned confusion is the fact that a review of experimental data on the viscosity of polyelectrolyte solutions shows large inconsistencies. ${ }^{4,5} \mathrm{It}$ is only recently that the reason for this discrepancy between different experimental measurements has been established. In careful measurements of the shear rate dependence of the viscosity, Boris and Colby ${ }^{4}$ and Krause et al. ${ }^{5}$ showed that polyelectrolyte solutions were shear thinning at extremely low shear rates, and argued that most of the older experiments did not report the relevant viscosity in the low shear limit.

In the last ten years, several theories studies have been put forward. Notable among them are the mode coupling theory calculation by Borsali et al. ${ }^{6}$ and by Rabin et al., ${ }^{3}$ the scaling theory of Dobrynin et al., ${ }^{7}$ the effective medium theory of Muthukumar, ${ }^{8}$ and the Kirkwood theory of Nishida et al. ${ }^{9}$ The scaling theory does not predict a peak in the reduced viscosity, in salt-free solutions. The theory of Muthukumar argues that the peak in $\eta_{R}$ arises from a screening of intramolecular hydrodynamic interactions as the concentration is increased. The theories of Borsali et al. ${ }^{6}$ Rabin et al. ${ }^{3}$ and Nishida et al. ${ }^{9}$ predict that the peak in $\eta_{R}$ arises due to increased screening from counterions as the concentration is increased, and are similar in spirit to the modecoupling theory for charged colloids ${ }^{10}$ which is argued to be accurate for the viscosity of spherical polyelectrolytes. ${ }^{11}$ Other theories rely on conformational changes of polymers with changing concentration. These theories do not take into account the interesting behavior in the static structure factor in dilute polyelectrolyte solutions. 
The basic idea of the present work is that the features in $\eta_{R}$ arise from the behavior of the static structure factor of dilute and semidilute polyelectrolyte solutions. In dilute solutions, the static structure factor displays a prominent peak at low wave vectors. ${ }^{12,13}$ As the concentration is increased the peak broadens and moves to higher wave vectors (see Fig. 2 and discussion in Sec. III). This indicates the presence of strong liquid like correlations on long length scales at low concentrations; correlations that become less important as the concentration is increased. This observation naturally leads to the question: Could this strong non monotonic concentration dependence of static pair correlations be the main physics behind the anomalous behavior of the viscosity? To this end, we develop a liquid state theory that incorporates the behavior of the static structure factor of polyelectrolyte solutions. While it has been suggested ${ }^{14,15}$ that the interesting concentration dependence of viscosity in dilute polyelectrolyte solutions could arise from the intermolecular pair correlations (as reflected in the peak in the structure factor at small wave numbers), the relationship between the viscosity and the structure factor is by no means obvious. It is of interest, therefore, to develop a quantitative theory relating the static structure to the viscosity.

We consider a system of charged rods and present a selfconsistent mode-coupling theory for the viscosity. We choose to study rods in order to focus on intermolecular effects. There have been many theories that explain the concentration dependence of the reduced viscosity on intramolecular effects. Studying a system with rigid molecules allows us to isolate intermolecular effects since conformational changes and intramolecular interactions are absent. Although we are not aware of experiments for the viscosity of rodlike polyelectrolytes, these are certainly possible, for example on solutions of tobacco mosaic virus (TMV) particles.

We present a self-consistent mode-coupling theory for the viscosity of unentangled polyelectrolyte solutions. Starting with the polymer center-of-mass density as the slow variable, we develop an expression for the polymer contribution to the viscosity that is identical to that of Geszti. ${ }^{16}$ The intermediate scattering function is obtained from the selfconsistent mode coupling theory as in the approach of Götze and co-workers. ${ }^{17}$ The center-of-mass structure factor, required in the theory, is obtained approximately from integral equation theory. ${ }^{18-20}$

The theory explains the behavior of the viscosity based purely on the behavior of the static structure factor. For short chains, the theory predicts that in salt-free solutions $\eta_{R}$ decreases monotonically with increasing concentration. With small amounts of added salt $\eta_{R}$ displays a shallow peak as a function of concentration at low concentrations. For longer chains, the theory predicts a peak in $\eta_{R}$ as a function of concentration for all salt concentrations, including salt-free solutions. The peak occurs at concentrations slightly lower than the overlap threshold concentration $c^{*}$. With the addition of salt, the intensity of the peak diminishes, but the position is unchanged. For a given salt concentration, the height of the peak increases dramatically as the degree of polymerization is increased, but the position is unchanged. These predictions can be tested experimentally.
The rest of this paper is organized as follows: Section II outlines the theory, Sec. III presents some results and a discussion, and Sec. IV presents some conclusions.

\section{MODE-COUPLING THEORY}

The polyelectrolyte solution consists of charged rodlike polymers and counterions. Each rod consists of $N$ tangent charged hard spheres of diameter $\sigma$; counterions are charged hard spheres of diameter $\sigma$. We combine a recently developed quantitatively accurate theory for the liquid structure in charged interacting polyelectrolytes ${ }^{18-20}$ with a selfconsistent mode coupling theory (MCT) to study the dynamics. The starting point for the calculation of viscosity $\eta$ is the Green-Kubo formula ${ }^{21}$

$$
\eta=\lim _{k \rightarrow 0} \frac{1}{k_{\mathrm{B}} T V} \int_{0}^{\infty} d t\left\langle\sigma^{z x}(k, t) \sigma^{z x}(-k, 0)\right\rangle,
$$

where $k_{\mathrm{B}}$ is Boltzmann's constant, $T$ is the temperature, $V$ is the volume, $\sigma^{z x}(k, t)$ is the transverse (or off-diagonal) component of the wave vector $k$ and time $t$ dependent stress tensor, and $\langle\cdots\rangle$ denotes an average over an equilibrium ensemble. The total transverse stress tensor of a polyelectrolyte solution contains contributions from solvent, polymer, and small ions.

For dilute and semidilute polyelectrolyte solutions several simplifications are possible: First of all, the contribution of the solvent is simply given by the viscosity in the absence of the solute $\eta_{0}$. It is because the presence of low concentrations of polymer and electrolyte are not expected to alter the solvent dynamics. Second, there is a contribution of the rotational Brownian motion. ${ }^{22}$ Last, there is a contribution due to polymer-polymer interactions $\eta_{p-p}$ which is expected to dominate over the contributions from small ions. Therefore we argue that $\eta \approx \eta_{0}+\eta_{r}+\eta_{p-p}$ and focus on the calculation of the polymer contribution. $\eta_{r}$ is calculated by neglecting the effect of the interactions on the rotational degree of freedom and is given by ${ }^{22}$

$$
\eta_{r}=\frac{2}{15} \frac{c_{p}}{N} \zeta_{r}
$$

where $\zeta_{r}$ is the rotational friction coefficient and is evaluated by a hydrodynamic calculation of an ellipsoid of the aspect ratio $N$ as ${ }^{23}$

$$
\zeta_{r}=\frac{2 \pi \eta \sigma^{3}}{3} \frac{N^{4}-1}{\frac{2 N^{2}-1}{\sqrt{N^{2}-1}} \ln \left(N+\sqrt{N^{2}-1}\right)-N} .
$$

For $\eta_{p-p}$, we employ a mode coupling treatment similar to that of Geszti ${ }^{16}$ to derive a microscopic expression.

The first step in the mode coupling approach is the choice of the slow collective variables for the description of the dynamics of the required correlation functions. Natural choice is the hydrodynamic variables, i.e., the three momentum current densities of polyion, $J_{\alpha}(\mathbf{k})$, for the co-ordinates $\alpha=x, y$, and $z$, and the polyion number density $\rho_{P}(\mathbf{k})$ defined as $\rho_{P}(\mathbf{k})=\sum_{i=1}^{N_{P}} e^{-i \mathbf{k} \cdot \mathbf{r}_{i}}$ where $\mathbf{r}_{i}$ is the position of the center of mass of the $i$ th polyion and $N_{P}$ is the number of 
polyions. A calculable microscopic relation for the viscosity is obtained by using the projection-operator formalism to rewrite the well-known Green-Kubo time correlation function expression in terms of $P$ and $Q$ operators. ${ }^{21}$ The standard approximation in the mode-mode coupling expansion is to consider the subspace of various binary products of the basic slow variables. Among such binary products, the odd ones with respect to time inversion do not contribute to the viscosity, and only the even combinations can be retained. The two obvious choices of the binary product are the density-density term and the current-current term. The current terms are expected to decay much faster than the density term, due to the friction with the surrounding solvent molecules. Thus, we neglect this contribution. Finally, all fourparticle correlations are approximated as the product of twoparticle correlations. With the above approximations and simplifications, the final expression for the zero frequency viscosity is written in terms of the density correlation function of the polyion as

$\eta=\eta_{0}+\eta_{r}+\frac{k_{\mathrm{B}} T}{60 \pi^{2}} \int_{0}^{\infty} d k k^{4} \int_{0}^{\infty} d t\left\{\frac{S^{\prime}(k)}{S(k)}\right\}^{2}\left\{\frac{F(k, t)}{S(k)}\right\}^{2}$,

where $S(k)$ is the static (center-of-mass) structure factor of the polyions, $F(k, t)$ is the corresponding intermediate scattering function, $S^{\prime}(k)$ is the derivative of $S(k)$ with respect to $k$.

In order to evaluate the viscosity, we need the intermediate scattering function $F(k, t)$ for the polyions and it should also be evaluated using MCT. As discussed above, we again assume that dynamics of counterions and solvent molecules is decoupled from dynamics of polyion. Then, the equation for $F(k, t)$ is expressed in a closed form as ${ }^{17}$

$$
\begin{aligned}
\frac{\partial}{\partial t} F(k, t)= & -\frac{D_{0} k^{2}}{S(k)} F(k, t)-\int_{0}^{t} d t^{\prime} M\left(k, t-t^{\prime}\right) \\
& \times \frac{\partial}{\partial t^{\prime}} F\left(k, t^{\prime}\right),
\end{aligned}
$$

where $D_{0}$ is the bare collective diffusion coefficient. $M(k, t)$ is the memory kernel given by

$M(k, t)=\frac{\rho_{P} D_{0}}{2} \int \frac{d \mathbf{q}}{(2 \pi)^{3}} V_{\mathbf{k}}^{2}(\mathbf{q}, \mathbf{k}-\mathbf{q}) F(|\mathbf{k}-\mathbf{q}|, t) F(q, t)$,

where $\rho_{P} \equiv\left\langle\rho_{P}(\mathbf{k}=\mathbf{0})\right\rangle=c_{p} / N$ is the average number density of polyion and $V(\mathbf{q}, \mathbf{k}-\mathbf{q})$ is the vertex function given in terms of the direct correlation function $c(q)$ as

$$
V(\mathbf{q}, \mathbf{k}-\mathbf{q})=\hat{\mathbf{k}} \cdot \mathbf{q} c(q)+\hat{\mathbf{k}} \cdot(\mathbf{k}-\mathbf{q}) c(|\mathbf{k}-\mathbf{q}|) .
$$

Equation (2.5) is a standard MCT equation familiar in the supercooled liquids and colloids ${ }^{17,24,25}$ community. This equation is a nonlinear integro-differential equation which has to be solved self-consistently. The numerical procedure to solve Eq. (2.5) is elucidated in Ref. 24.

The bare diffusion coefficient $D_{0}$ is obtained from the value for a long ellipsoid calculated from hydrodynamic cal- culations using stick boundary conditions. For the ellipsoid with the aspect ratio of $N$, the total diffusion coefficient is given $b^{23}$

$D_{0}=\frac{D_{\|}+2 D_{\perp}}{3}=\frac{k_{\mathrm{B}} T}{3 \pi \eta_{0} \sigma} \frac{1}{\sqrt{N^{2}-1}} \ln \left(N+\sqrt{N^{2}-1}\right)$.

Note that this theory considers only the translational motion of the rods, which is assumed to be isotropic. We argue that we can neglect the anisotropy in translation and its coupling to rotation in the concentration regimes we consider. We estimate the contribution from the anisotropy and coupling to diffusion as follows: In the dilute limit, if the interaction between polyions is neglected, it is possible to solve the rotation-coupled diffusion equation and evaluate $F(k, t)$ exactly. ${ }^{22,26-29}$ If the ratio between the parallel $\left(D_{\|}\right)$and perpendicular $\left(D_{\perp}\right)$ diffusion coefficients is not very large, the change in the relaxation rate of $F(k, t)$ at short times arising from a coupling with rotational diffusion is also small. For example, for $D_{\|} / D_{\perp} \simeq 2$, the relaxation rate of $F(k, t)$ at short times is changed by less than $10 \%$ due to the coupling with the rotational diffusion. At longer times, $t \geqslant \tau_{R}$, where $\tau_{R}$ is the rotational relaxation time, $F(k, t)$ is simply given by $\exp \left[-D_{0} k^{2} t\right]$, where $D_{0}$ is the average diffusion coefficient defined by $D_{0}=\left(D_{\|}+2 D_{\perp}\right) / 3$. Thus, decoupling rotation from translation and assuming the translation diffusion is isotropic are reasonable approximations in dilute solutions. These approximations become questionable when the concentration or rod length becomes large when, due to the entanglement effects, the rotational time increases steeply and anisotropy of the translational diffusion will be enhanced. This regime, however, is far beyond the scope of the present paper. ${ }^{30}$

\section{STATIC PROPERTIES}

To proceed further we require a model for the polyions and a means of calculating the static structure of the polyion centers of mass. In this work the molecules are modeled as a collection of interaction sites arranged linearly in a rodlike configuration. Each particle consists of $N$ tangent charged hard spheres (or sites) with hard sphere diameter $\sigma$, which is used as the unit of length in this paper. Each sphere carries a negative fractional charge $f e$, where $e$ is the charge on an electron. The effect of solvent and small ions is included into the potential of interaction between sites on the polyelectrolyte molecules. The resulting effective potential $\beta u(r)$ is given by

$$
\beta u(r)=\left\{\begin{array}{l}
\infty \quad \text { for } r<\sigma \\
\Gamma \exp (-\kappa r) / r \quad \text { for } r>\sigma,
\end{array}\right.
$$

where $\beta=1 / k_{\mathrm{B}} T, \quad \Gamma=f^{2} l_{B} /(1+\kappa \sigma), \quad l_{B} \equiv \beta e^{2} / \varepsilon \quad$ is the Bjerrum length, $\varepsilon$ is the dielectric constant of the solvent, and $\kappa$ is the inverse screening length, $\kappa$ $=\sqrt{4 \pi l_{B}\left(f^{2} c_{p}+2 c_{s}\right)}$ where $c_{s}$ is the number density of the (monovalent) salt, and $c_{p}$ is the number density of polymer sites. In all the calculations presented in this work, $l_{B}$ $=0.758 \sigma$ and $f=1$. If $\sigma \approx 4 \AA$, then an added salt concentration of $1 \mathrm{mM}$ corresponds to a reduced salt concentration of $c_{s} \sigma^{3} \approx 4 \times 10^{-5}$. 


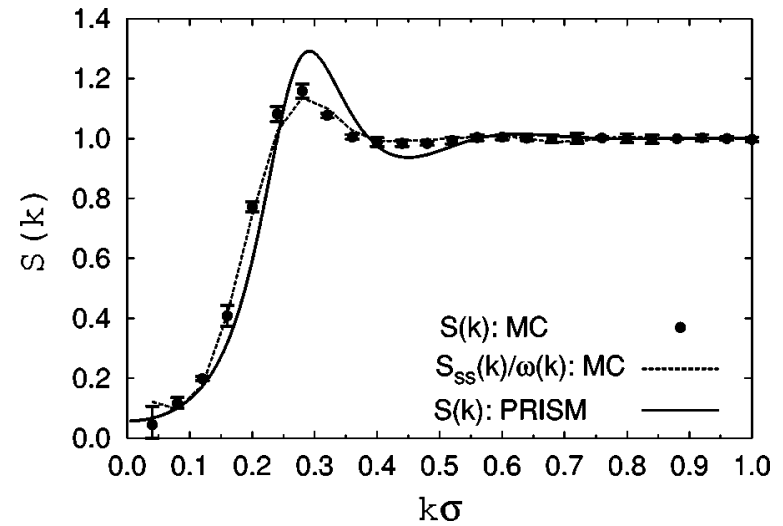

FIG. 1. Comparison of PRISM predictions (solid lines) for the center-ofmass structure factor to Monte Carlo simulation results for $c_{p} \sigma^{3}=10^{-3}$, $l_{B}=\sigma$, and $N=20$. Dashed lines are simulation results for $S_{s s}(k) / \hat{\omega}(k)$.

The center-of-mass static structure factor is calculated using integral equations. The single chain structure factor $\hat{\omega}(k)$ is known exactly for this model. The site-site static structure factor $S_{s s}(k)$ is obtained from the polymer reference interaction site model (PRISM) theory, ${ }^{31}$ as described elsewhere. ${ }^{19}$ It has previously been established, ${ }^{32}$ by direct comparison of theoretical predictions for $S_{s s}(k)$ to computer simulations that PRISM is accurate for $S_{s s}(k)$. The centerof-mass structure factor is the approximated as $S(k)$ $\approx S_{s s}(k) / \omega(k)$. To check the validity of this approximation, we perform Monte Carlo simulations of rods interacting via screened Coulomb interactions, and calculate $S_{s s}(k)$ and $S(k)$. The simulation algorithm is identical to that described elsewhere ${ }^{32}$ except that we do not perform the Ewald sum. Figure 1 compares simulations results for $S(k)$ (filled circles) and $S_{s s}(k) / \omega(k)$ (dotted lines) for $l_{B}=\sigma, c_{p} \sigma^{3}=10^{-3}$, and $N=20$, and shows that the approximation for $S(k)$ is quite accurate. Also shown in the figure is the PRISM prediction for $S(k)$. The PRISM $S(k)$ correctly reproduces the liquidlike structure manifested in the peak of $S(k)$. In fact, the theory is in quantitative agreement with the simulation results.

Figure 2 depicts $S(k)$ from PRISM for $N=150$ and for several polyion concentrations. We also show the results derived from a simpler Debye-Huckel (DH) approximation. The DH result is derived by taking the $\sigma \rightarrow 0$ limit, and approximating the site-site direct correlation function, $c_{s s}(r)$, by $c_{s s}(r)=-\beta u(r)$ for all $r$. The resulting intermolecular structure factor, denoted $S_{\mathrm{DH}}(k)$, is given by

$$
S_{\mathrm{DH}}(k)=\frac{1}{1+\frac{4 \pi l_{B}}{k^{2}+\kappa^{2}} c_{p} \omega(k)} .
$$

\section{RESULTS}

For salt-free solutions, the theory predicts that the reduced viscosity $\eta_{R}$ is a monotonically decreasing function of polymer concentration, for short chains. As the chain length is increased, a peak in $\eta_{R}$ is predicted, at concentrations slightly below the overlap threshold concentration. Figures 3(a) and 3(b) depict $\eta_{R}$ as a function of polymer concentra-

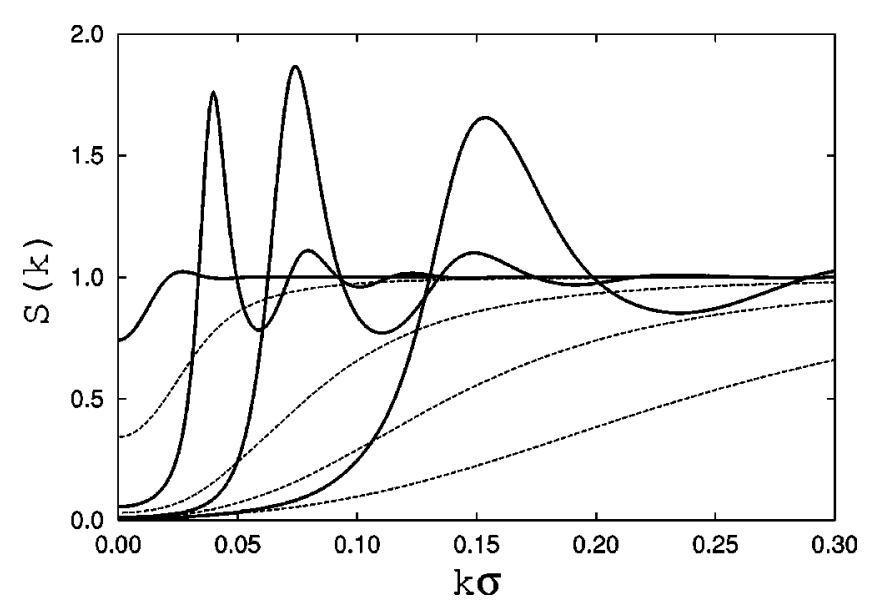

FIG. 2. Static structure factor $S(k)$ predicted using the PRISM theory (solid lines) and the $\mathrm{DH}$ approximation (dotted lines) for $N=150$ and $c_{s}$ $=1 \mathrm{mM}$, and for various polyion concentrations. From left to right, the polyion concentrations are $c_{p} \sigma^{3}=10^{-6}, 2 \times 10^{-5}, 10^{-4}$, and $5 \times 10^{-4}$.

tion for various concentrations of added salt, and for degrees of polymerization of $N=20$ and 150 , respectively. (The added salt concentrations of 1,2 , and $5 \mathrm{mM}$ correspond to reduced salt concentrations of $c_{s} \sigma^{3} \approx 4 \times 10^{-5}, 8 \times 10^{-5}$, and $2 \times 10^{-4}$, respectively.) In the figures, the abscissa is the polymer concentration divided by the overlap threshold concentration $c^{*}$ which, for this model, is given by $c^{*} \sigma^{3}$ $=1 / N^{2}$. In all cases we find that the major contribution comes from the polymer-polymer interaction given by the mode-coupling expression in Eq. (2.4). The contribution of the rotational Brownian motion of the individual $\operatorname{rod} \eta_{r}$ is independent of the polyion density and does not affect the qualitative behavior except for the low concentration regime where the mode-coupling contribution becomes very small.

For $N=20$ [Fig. 3(a)] and $c_{s}=0$ (salt-free), $\eta_{R}$ is a monotonically decreasing function of $c_{p} / c^{*}$. As the salt concentration is increased, the value of $\eta_{R}$ decreases at all polymer concentrations. For $N=20$ this results in a shallow peak in $\eta_{R}$ at low polymer concentrations and $1 \mathrm{mM}$ salt. For high values of added salt $\eta_{R}$ is a monotonically increasing function of polymer concentration. These results are typical of cases when the static structure factor does not display a very strong peak at low wave vectors. The influence of the longrange liquidlike order on the dynamic properties is therefore very weak. The predictions for short chains are qualitatively similar to other theories ${ }^{6,9}$ that ignore the effect of static structure on the dynamic properties.

As the degree of polymerization $N$ is increased, the theory predicts a prominent peak in $\eta_{R}$ that occurs at a concentration just below the overlap threshold concentration. The amplitude of this peak increases with increasing degree of polymerization and decreases with increasing salt concentration. This can be seen in Fig. 3(b) which depicts $\eta_{R}$ as a function of $c_{p} / c^{*}$ for $N=150$. In salt-free solutions, the peak in $\eta_{R}$ is very prominent. The addition of salt dramatically reduces the height of the peak, although a peak is clearly present even for high $(5 \mathrm{mM})$ salt concentrations. (Note that both axes are plotted on a logarithmic scale in order to fit all the curves on the same figure.) 

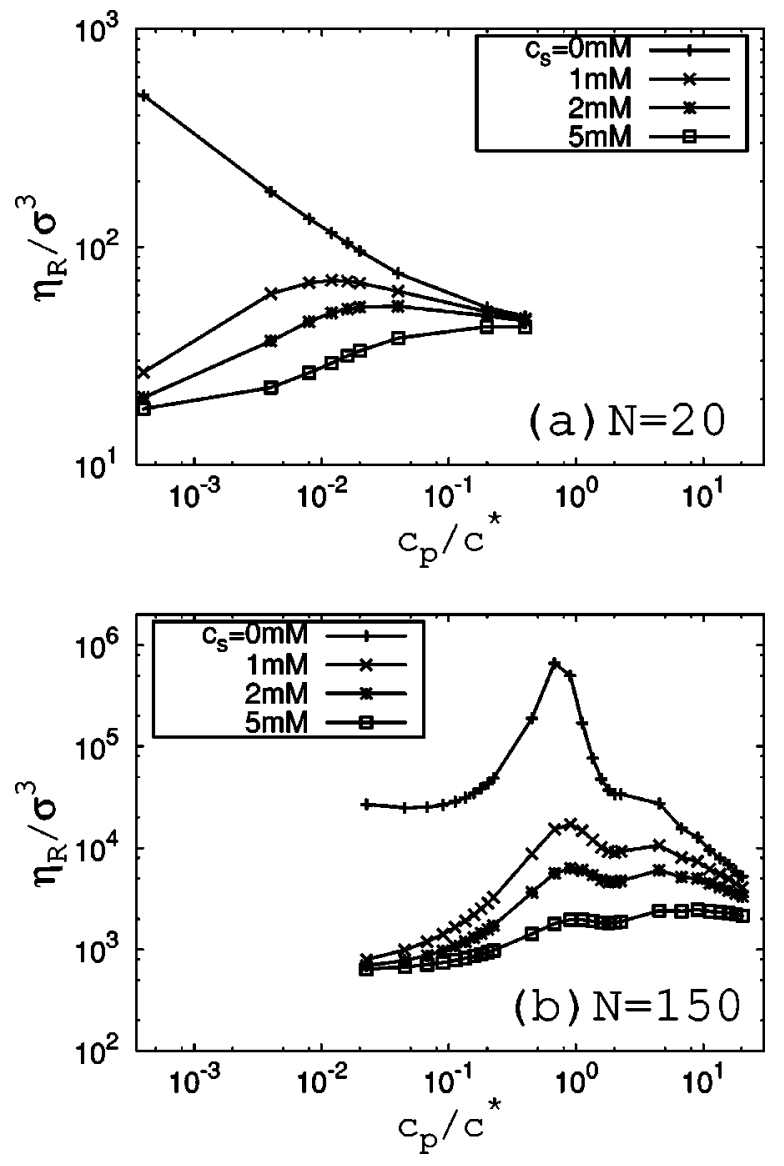

FIG. 3. Dependence of the reduced viscosity $\eta_{R}$ on polymer concentration for various salt concentrations, $c_{s}=0 \mathrm{mM}(+), 1 \mathrm{mM}(\times), 2 \mathrm{mM}(*)$, and $5 \mathrm{mM}(\square)$, and for (a) $N=20$ and (b) $N=150$. Note that the abscissa is concentration divided by the overlap threshold concentration $c^{*}$, and both axes are logarithmic.

The reduced viscosity is a strong function of chain length, in a manner that depends on the salt concentration. Figures 4(a) and 4(b) depict $\eta_{R}$ as a function of polymer concentration for various values of $N$ and $c_{s}=1$ and $2 \mathrm{mM}$, respectively. In both salt-free and added salt solutions, the peak in $\eta_{R}$ grows with increasing degree of polymerization, but the position of the peak is insensitive to the value of $N$. Figure 5 depicts the value of $\eta_{R}$ at the maximum as a function of degree of polymerization for various salt concentrations. The peak values is fitted well by a power law except for the salt-free case. The molecular weight dependence is very strong, much stronger than what is obtained for entangled (neutral) polymer melts. The exponent decreases dramatically as the salt concentration is increased.

The physical interpretation of these results is that the peak in $\eta_{R}$ arises from intermolecular correlations between the rods. The main physical feature that is input into the theory is an accurate estimate of the static structure factor of the polyelectrolyte solutions. The viscosity is then calculated using a fully self-consistent mode-coupling theory. Any scaling analysis of the dependence of $\eta_{R}$ on $N$ and $c_{p}$ must take into account the complex dependence of static correlations on the dynamics.
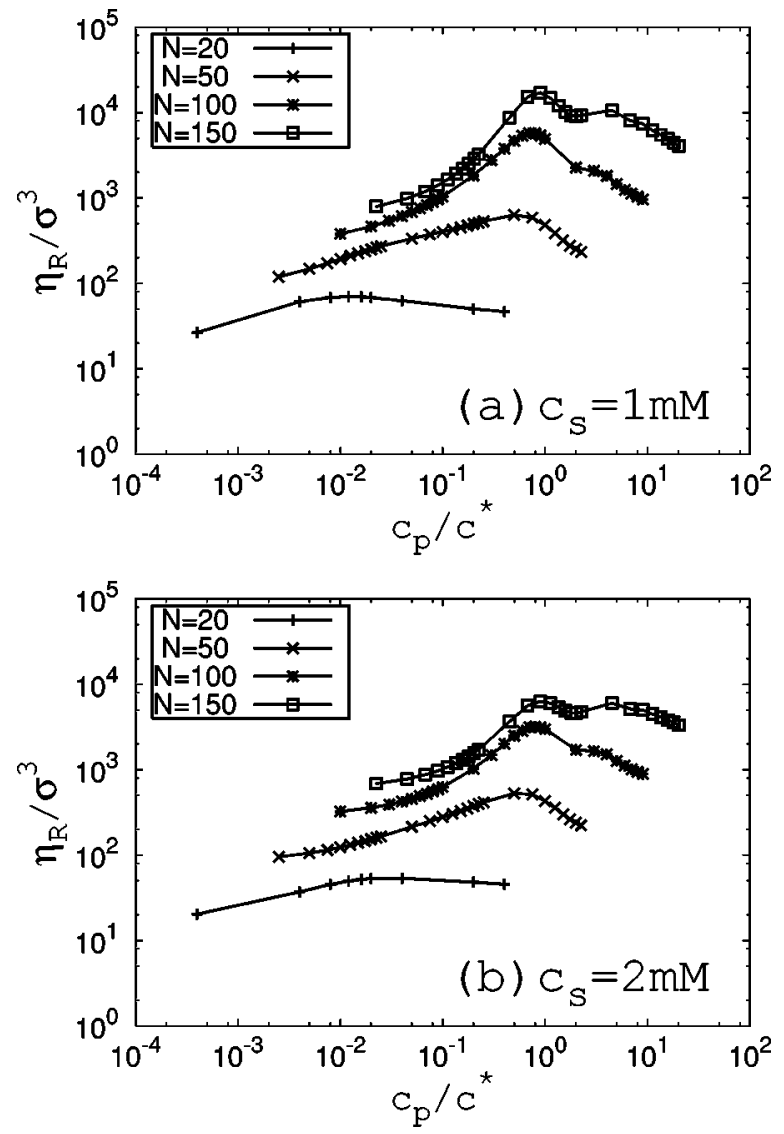

FIG. 4. Dependence of the reduced viscosity $\eta_{R}$ on polymer concentration for various degrees of polymerization, $N=20(+), 50(\times), 100\left(^{*}\right)$, and 150 ( $\square$ ), and for (a) $c_{s}=1 \mathrm{mM}$ and (b) $c_{s}=2 \mathrm{mM}$. Note that the abscissa is concentration divided by the overlap threshold concentration $c^{*}$ and both axes are logarithmic.

\section{DISCUSSION}

The main ingredients of the theory of this work are (i) the use of a fully self-consistent mode-coupling approximation (SCMCT), and (ii) accurate estimates of the structure of the solution. It is of interest to determine how the actual predictions depend on these two components. We compare

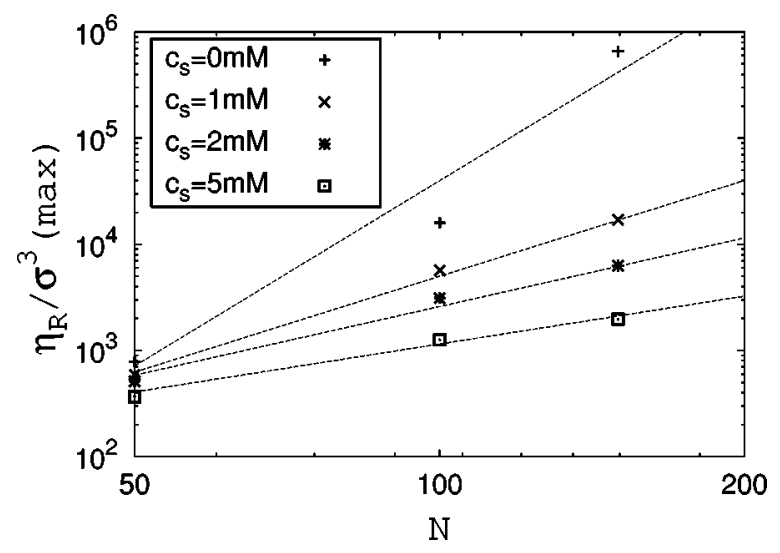

FIG. 5. $N$ dependence of the peak value of $\eta_{R}$ for various salt concentrations. Dotted lines are power law fits $N^{\nu}$, with $\nu=5.8,3,2.2$, and 1.5, for $c_{s}=0,1,2$, and $5 \mathrm{mM}$, respectively. 


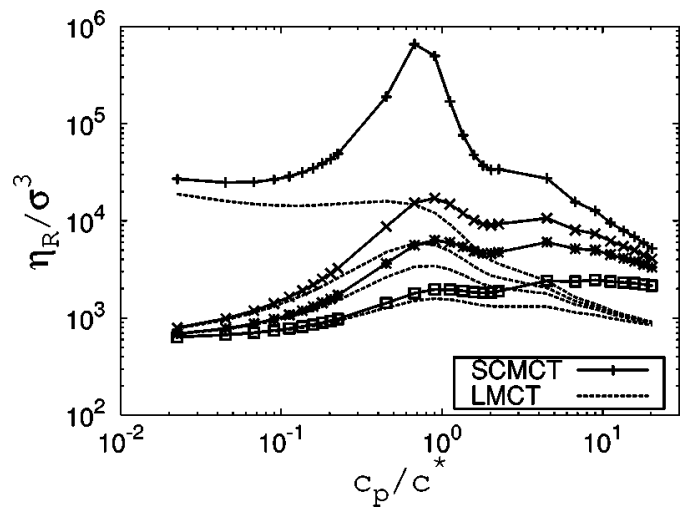

FIG. 6. Comparison of predictions for the concentration dependence of $\eta_{R}$ from a fully self-consistent MCT (SCMCT) (-) and lowest order MCT (LMCT) $(---)$ for $N=150$. PRISM results for $S(k)$ are used in all calculations. In each case, the curves correspond to (from top to bottom) salt concentrations of $c_{s}=0 \mathrm{mM}(+), 1 \mathrm{mM}(\times), 2 \mathrm{mM}(*)$, and $5 \mathrm{mM}(\square)$.

the predictions of this work with those of related, and simpler, theoretical schemes, as described below.

\section{A. Comparison of SCMCT with lowest order MCT (LMCT)}

The MCT requires an expression for the intermediate scattering function $F(k, t)$, which we obtain from the selfconsistent mode coupling equation, Eq. (2.5). A simpler approximation amounts to neglecting the memory kernel $M(k, t)$ in Eq. (2.5). We refer to this approximation, where $F(k, t)$ is simply given by

$$
F(k, t)=S(k) \exp \left[-\frac{D_{0} k^{2} t}{S(k)}\right]
$$

as the lowest order MCT (LMCT). Such an approximation has been previously investigated by others ${ }^{6,3}$ but with the Debye-Hückel (DH) approximation for the static structure. The theory of Nishida et al. ${ }^{9}$ is closely related to the LMCT with an approximate (concentration independent) structure factor obtained from numerical calculations at zero density. In the LMCT, Eq. (2.4) is readily integrated over time to give

$$
\eta_{R, \mathrm{LMCT}}=\frac{k_{\mathrm{B}} T}{120 \pi^{2} \eta_{0} c_{p} D_{0}} \int_{0}^{\infty} d k k^{2} \frac{\left[S^{\prime}(k)\right]^{2}}{S(k)} .
$$

Figure 6 compares predictions from the SCMCT (solid lines) and LMCT (dashed lines) for the concentration dependence of $\eta_{R}$ for $N=150$. PRISM predictions for $S(k)$ are used in both cases. The qualitative behavior predicted by both theories is similar, i.e., there is a peak in $\eta_{R}$ at concentrations of the order of the overlap threshold concentration, and the amplitude of the peak diminishes as the concentration of added salt increases. The position of this peak is insensitive to concentration of added salt and degree of polymerization. The quantitative differences, however, are enormous, with SCMCT predicting a value of $\eta_{R}$ at the peak that is two orders of magnitude higher than LMCT. This emphasizes the fact that memory effects are of considerable importance in these systems. This difference between LMCT and SCMCT grows with increasing chain length. For example the theories are almost indistinguishable for $N=20$.

\section{B. Influence of static structure}

In order to see how the accurate estimates of the structure affects the results, we compare MCT predictions for the viscosity using PRISM results for the structure factor to those using the DH approximation for $S(k)$ given by Eq. (3.2). A good test of the importance of liquid structure would be to compare SCMCT with PRISM $S(k)$ to SCMCT with the DH $S(k)$. We find, however, that SCMCT with the DH $S(k)$ predicts a so-called ergodic-nonergodic transition, i.e., $F(k, t)$ fails to relax to zero, for low polyion concentrations. Such a transition, which is also predicted by the MCT for supercooled liquids, leads to a divergence in the viscosity at finite concentrations! This prediction is clearly incorrect, since no such divergence is seen in experiment, or expected on physical grounds. This emphasizes, however, that SCMCT is very sensitive to the structure factor used as input, as one would expect. We attribute the fictitious transition to an overestimation of the memory kernel of Eq. (2.6) at large wave vectors which allows anomalous positive feedback into the relaxation of $F(k, t)$. This over estimation of the memory kernel arises from the broadened and featureless $S(k)$ in the DH approximation. In reality, the hard-core interaction comes into play at large $k$, thus resulting in a flattening of $S(k)$. The hard-core interaction is, of course, neglected in the DH approximation.

We therefore investigate the influence of structure using the LMCT. A combination of the DH and LMCT approximations allows us to derive simple scaling results for the viscosity. Since the form factor $\omega(k)$ depends on $k$ only weakly, we can set $\omega(k) \approx \omega(0)=N$ in Eq. (3.2) to get

$$
\begin{aligned}
S(k) & \approx \frac{1}{1+\frac{4 \pi l_{B} N c_{p} \sigma^{3}}{k^{2}+\kappa^{2}}} \\
& =\frac{1}{1+A \alpha(x)},
\end{aligned}
$$

where $x=k / \kappa, A=4 \pi l_{B} N c_{p} \sigma^{3} / \kappa^{2}=N c_{p} \sigma^{3} /\left(c_{p}+2 c_{s}\right)$, and $\alpha(x)=1 /\left(1+x^{2}\right)$. With these simplifications, $\eta_{R}$ from the LMCT is given by

$$
\eta_{R}=\frac{k_{B} T A^{2} \kappa}{120 \pi^{2} \eta_{0} c_{p} \sigma^{3} D_{0}} \int_{0}^{\infty} d x x^{2} \frac{\left[\alpha^{\prime}(x)\right]^{2}}{(1+A \alpha)^{2}},
$$

where we have neglected the rotation contribution $\eta_{r}$, which does not affect the argument.

If we ignore the concentration dependence of the integral, which is expected to be weak, then

$$
\eta_{R} \sim \frac{N^{2} c_{p} l_{B}^{2}}{D_{0} \kappa^{3}} \sim \frac{N^{2} \sqrt{l_{B}}}{D_{0}} c_{p}\left(c_{p}+2 c_{s}\right)^{-3 / 2} .
$$

For salt-free solutions, this simple scaling approach predicts $\eta_{R} \sim 1 / \sqrt{c_{p}}$, which is the same as the Fuoss Law or the scaling theory of Rubinstein and co-workers. ${ }^{7}$ In solutions with added salt, this scaling approach predicts a peak in $\eta_{R}$ as a function of $c_{p}$. The peak occurs for $c_{p}=4 c_{s}$, independent of any of the other parameters.

Figure 7 compares predictions for $\eta_{R}$ using the LMCT, and DH and PRISM approximations for $S(k)$. The predic- 


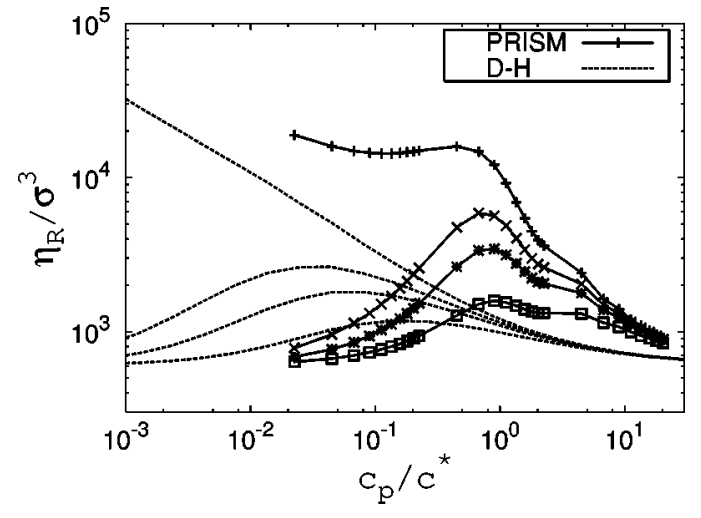

FIG. 7. Comparison of predictions for the concentration dependence of $\eta_{R}$ from lowest order MCT using PRISM input for $S(k)(-)$ and lowest order MCT using Debye-Hückel input for $S(k)(---)$ for $N=150$. In each case, the curves correspond to (from top to bottom) salt concentrations of $c_{s}=0 \mathrm{mM}(+), 1 \mathrm{mM}(\times), 2 \mathrm{mM}(*)$, and $5 \mathrm{mM}(\square)$.

tions of LMCT with DH $S(k)$ is drastically different from that with PRISM $S(k)$. For one, no peak in $\eta_{R}$ is observed in the DH theory in salt-free solutions. A peak appears with added salt, but this peak is very broad compared to the predictions of the theory with PRISM input. The position of the peak moves to smaller concentrations as the concentration of added salt is decreased. The predictions of LMCT with DH $S(q)$ is qualitatively similar to the scaling results, Eq. (5.5). The physical reason for the peak is completely different from SCMCT with PRISM and comes from a competition between the increase in $\eta_{R}$ with increasing concentration and the decrease in $\eta_{R}$ from decreasing the inverse screening length. The theories of Cohen et al., ${ }^{3}$ Borsali et al. ${ }^{6}$ and Nishida et al. ${ }^{9}$ are similar in spirit to the lowest order MCT with DH input.

\section{CONCLUSIONS}

We present a mode-coupling theory for the viscosity of dilute polyelectrolyte solutions. Using the static structure factor from polyelectrolyte integral equation theory as input, we calculate the viscosity using a self-consistent modecoupling approach. The theory predicts a peak in the reduced viscosity for long enough polymer chains that occurs at concentrations smaller than the overlap threshold concentration. When the reduced viscosity is plotted against the polymer concentration divided by the overlap threshold concentration, the peak position is independent of degree of polymerization or added salt concentration, although the height of the peak increases strongly with increasing degree of polymerization and decreases strongly with increasing the concentration of added salt.

An important caveat is that this theory does not attempt to treat entanglement effects. The theory assumes that rotational and translational diffusion are decoupled, and that the anisotropy in translational diffusion can be ignored. These approximations are not expected to be important in dilute solutions but preclude the application of this theory to semidilute and concentrated solutions of polyelectrolytes. ${ }^{30}$

The theoretical predictions are sensitive to the level of approximation within the MCT and the structure factors used. If a lowest order MCT is employed, the theoretical predictions are qualitatively similar to the self-consistent MCT (SCMCT), but the value of the reduced viscosity is different by an amount that increases dramatically with chain length. If the Debye-Hückel (DH) approximation is used for the structure factor, SCMCT predicts a glass transition at low concentrations, which arises from the neglect of hard-core interactions. With the DH structure factor and lowest order MCT (LMCT), the theory predicts peaks in the reduced viscosity with polymer concentration, but the position and amplitude of these peaks are different from what is observed in the full theory.

A rigorous comparison with experiment is not possible because detailed experiments on the viscosity of rodlike polyelectrolytes in good solvents are not available. We therefore compare theory to viscosity data of (poly)styrene sulfonate (PSS) dissolved in water. Additional complications in these systems are the possible importance of intramolecular effects due to chain flexibility and hydrophobic interactions. There are significant differences between theoretical predictions for rods and experiments on these flexible chains. In many experiments ${ }^{5}$ the peak in $\eta_{R}$ occurs in the semidilute regime, while the focus of theoretical work has been on such a peak in the dilute solution regime. The SCMCT does predict a second peak in $\eta_{R}$ in the semidilute regime (see, for example, Fig. 7) as well, but, strictly, this is outside the regime where we expect the theory to be accurate. In experiments on sodium PSS ${ }^{14}$ the peak in $\eta_{R}$ moves to higher concentrations as the molecular weight is increased. In the SCMCT for rods the peak position occurs at $c_{p}=c^{*}$ for all $N$, i.e., the peak positions moves drastically to lower concentrations as $N$ is increased. We carry out the MCT calculation in the same fashion but with the center-of-mass structure factor for flexible chains and find that it gives essentially similar results as rods and does not explain the $N$ dependence of $\eta_{R}$. This is clearly due to the neglect of all other slow modes that originate from the intramolecular configuration appearing in the case of flexible polymers. Muthukumar ${ }^{8}$ makes a similar prediction. The colloidlike theories, including those considered by Cohen et al. ${ }^{3}$ and Antonietti et al.,${ }^{14}$ predict that the peak is independent of $N$, and in fact occurs for a concentration $c_{p}=4 c_{s}$, which is not in agreement with experiment on PSS. Therefore none of the theories can claim to explain experimentally observed results, and the occurrence of the peak in $\eta_{R}$ must still be considered anomalous.

In conclusion, we present a fully self-consistent modecoupling theory for the viscosity of rodlike polyelectrolyte solutions. Significant differences are present between the predictions of the theory and experiments for flexible chains. Measurements on theoretically well understood systems such as solutions of TMV will therefore be very useful as a test of the theory. Note that since the only input into the theory is the static structure factor, which has been measured in TMV solutions, this theory can be used to make parameter-free predictions for the absolute viscosity in those systems. We hope this work will stimulate further experimental and computational work on this problem. 


\section{ACKNOWLEDGMENTS}

We thank Professor Mathias Fuchs for assistance with the numerical solution of the MCT equations. K.M. acknowledges support from the Japan Society for the Promotion of Science (JSPS). A.Y. thanks the Jawaharlal Nehru Center for Advanced Research for support and hospitality during his stay in Bangalore, and Professor Ralph Colby for useful discussions. This material is based upon work supported by the National Science Foundation under Grant No. CHE-0315219 (to A.Y.). B.B. thanks Department of Science and Technology for support.

${ }^{1}$ S. Förster and M. Schmidt, Adv. Polym. Sci. 120, 51 (1995).

${ }^{2}$ H. Vink, Polymer 33, 3711 (1992).

${ }^{3}$ J. Cohen, Z. Priel, and Y. Rabin, J. Chem. Phys. 88, 7111 (1988).

${ }^{4}$ D. C. Boris and R. H. Colby, Macromolecules 31, 5746 (1998)

${ }^{5}$ W. E. Krause, J. S. Tan, and R. H. Colby, J. Polym. Sci., Part B: Polym. Phys. 37, 3429 (1999).

${ }^{6}$ R. Borsali, T. A. Vilgis, and M. Benmouna, Macromolecules 25, 5313 (1992).

${ }^{7}$ M. Rubinstein, R. H. Colby, and A. V. Dobrynin, Phys. Rev. Lett. 73, 2776 (1994); A. V. Dobrynin, R. H. Colby, and M. Rubinstein, Macromolecules 28, 1859 (1995).

${ }^{8}$ M. Muthukumar, J. Chem. Phys. 105, 5183 (1996).

${ }^{9}$ K. Nishida, K. Kaji, and T. Kanaya, Polymer 42, 8657 (2001).

${ }^{10}$ W. Hess and R. Klein, Adv. Phys. 32, 173 (1983).

${ }^{11}$ M. Antonietti, A. Briel, and F. Gröhn, Macromolecules 33, 5950 (2000).

${ }^{12}$ E. E. Maier, S. F. Schulz, and R. Weber, Macromolecules 21, 1544 (1988).

${ }^{13}$ E. E. Maier, R. Krause, M. Deggelmann, M. Hagenbüchle, R. Weber, and S. Fraden, Macromolecules 25, 1125 (1992).
${ }^{14}$ M. Antonietti, A. Briel, and S. Förster, J. Chem. Phys. 105, 7795 (1996).

${ }^{15}$ St. Batzill, R. Luxemburger, R. Deike, and R. Weber, Eur. Phys. J. B 1, 491 (1998).

${ }^{16}$ T. Geszti, J. Phys. C 16, 5805 (1983).

${ }^{17}$ T. Franosch, M. Fuchs, W. Götze, M. R. Mayr, and A. P. Singh, Phys. Rev. E 55, 7153 (1997).

${ }^{18}$ A. Yethiraj and C.-Y. Shew, Phys. Rev. Lett. 77, 3937 (1996).

${ }^{19}$ C.-Y. Shew and A. Yethiraj, J. Chem. Phys. 106, 5706 (1997).

${ }^{20}$ A. Yethiraj, Phys. Rev. Lett. 78, 3789 (1997).

${ }^{21}$ U. Balucani and M. Zoppi, Dynamics of the Liquid State (Clarendon, Oxford, 1994), Chap. 6; and references therein.

${ }^{22}$ M. Doi and S. F. Edwards, The Theory of Polymer Dynamics (Clarendon, Oxford, 1986).

${ }^{23}$ J. Happel and H. Brenner, Low Reynolds Number Hydrodynamics (Kluwer Academic, Dordrecht, 1963).

${ }^{24}$ M. Fuchs, W. Götze, I. Hofacker, and A. Latz, J. Phys.: Condens. Matter 3, 5047 (1991); Th. Voigtmann, I. Hofacker, W. Götze, and M. Fuchs (unpublished)

${ }^{25}$ A. J. Banchio, G. Nägele, and J. Bergenholtz, J. Chem. Phys. 113, 3381 (2000).

${ }^{26} \mathrm{~K}$. Miyazaki (unpublished).

${ }^{27}$ T. Maeda and S. Fujime, Macromolecules 17, 1157 (1984).

${ }^{28} \mathrm{~K}$. Kubota, H. Urabe, Y. Tominaga, and S. Fujime, Macromolecules 17, 2096 (1984)

${ }^{29}$ In Refs. 27 and 28, they calculated the experimentally observed scattering function which is different from $F(k, t)$ used in this paper in that it includes the form factor in the correlation function. For our purpose, we simply left out this factor in their calculation.

${ }^{30}$ K. Miyazaki and A. Yethiraj, J. Chem. Phys. 117, 10448 (2002).

${ }^{31}$ K. S. Schweizer and J. G. Curro, Phys. Rev. Lett. 58, 246 (1987).

${ }^{32}$ C.-Y. Shew and A. Yethiraj, J. Chem. Phys. 110, 11599 (1999). 